\title{
A genotype-specific surgical approach for patients with Pfeiffer syndrome due to W290C pathogenic variant in FGFR2 is associated with improved developmental outcomes and reduced mortality
}

\author{
Tara L. Wenger, MD PhD ${ }^{1}$, Richard A. Hopper, MD², Anna Rosen, BS ${ }^{1}$, Hannah M. Tully, MD MSc ${ }^{3}$, \\ Michael L. Cunningham, MD PhD ${ }^{1}$ and Amy Lee, MD ${ }^{4}$
}

Purpose: Among children with FGFR2-associated Pfeiffer syndrome, those with the W290C pathogenic variant (PV) are reported to have the worst clinical outcomes. Mortality is high, and severe neurocognitive impairment has been reported in all surviving patients. However, it is unclear whether these poor outcomes are an unavoidable consequence of the PV itself, or could be improved with a genotype-specific treatment approach. The purpose of this report is to describe the more intensive surgical approach used for each of the three patients with W290C PV in FGFR2 at our center, all of whom survived and have normal neurocognitive functioning.

Methods: Retrospective chart review.

Results: In contrast to other patients with Pfeiffer syndrome at our center, all three patients who were subsequently found to have a W290C PV required a similar and more aggressive approach based on early cephalocranial disproportion. In contrast to previously reported W290C cases, each of our three patients survived and demonstrate normal neurocognitive functioning.

Conclusion: While previously reported outcomes in W290Cassociated Pfeiffer syndrome have been extremely poor, we present three patients who underwent an intensive surgical approach and have normal development. This suggests that a personalized and aggressive surgical approach for children with W290C PV may dramatically improve clinical outcome.

Genetics in Medicine (2019) 21:471-476; https://doi.org/10.1038/s41436018-0073-x

Key Words: Pfeiffer syndrome; Craniosynostosis; Cephalocranial disproportion; Neurodevelopment; Intracranial pressure

\section{INTRODUCTION}

Among children with FGFR2-associated craniosynostosis syndromes (i.e., Apert, Pfeiffer, Crouzon, Beare-Stevenson, Jackson-Weiss), children with the W290C pathogenic variant (PV) are reported to be the most severely affected. Approximately 20 children with this PV have been described in the literature, ${ }^{1-13} 7$ of whom died during childhood, primarily due to respiratory failure. ${ }^{4-7,9,12,13}$ Tracheal sleeves, which are associated with a $90 \%$ risk of sudden death by age 2 , were reported in all children with bronchoscopy and some were identified at autopsy. ${ }^{2-5,8,12-14}$ Of the 20 known patients, 10 had tracheal sleeve reported, an additional patient was reported to have "tracheal anomalies," 3 reported no tracheal anomalies, and the remaining 6 patient reports did not include documentation of tracheal anatomy. Among the six children reported by other groups surviving past 6 months of age, all references to development indicate each patient has had significant delays. At 15 years of age, the oldest surviving patient was only able to follow simple commands. ${ }^{10}$ The second oldest, at age 6, had a few signs and could sit independently. ${ }^{12}$

Cephalocranial disproportion (CCD) occurs when there is a mismatch between intracranial contents (brain and cerebrospinal fluid) and cranial volumetric space. ${ }^{15}$ In Pfeiffer syndrome, CCD commonly occurs because of multisuture craniosynostosis. If left untreated, CCD can cause damage to the developing brain and result in intellectual disability or even death. The surgical approach for CCD in multisuture craniosynostosis varies between institutions and there is no current national standard. Earlier protocols involved frontal expansion at an early age but had high rates of relapse. ${ }^{16}$ Subsequently, many groups moved toward posterior cranial vault expansion at age 3-9 months as the initial surgical procedure. ${ }^{17}$ At our center, each patient is evaluated as early as possible by our craniofacial team to determine the optimal timing and type of surgeries they will need. This evaluation includes urgent evaluation for CCD, medical comorbidities, review of imaging and coordination of surgical planning with

\footnotetext{
${ }^{1}$ Division of Craniofacial Medicine, Seattle Children's Hospital, Seattle, Washington, USA; ${ }^{2}$ Division of Craniofacial and Plastic Surgery, Seattle Children's Hospital, Seattle, Washington, USA; ${ }^{3}$ Division of Neurology, Seattle Children's Hospital, Seattle, Washington, USA; ${ }^{4}$ Division of Neurosurgery, Seattle Children's Hospital, Seattle, Washington, USA. Correspondence: Tara L. Wenger (Tara.wenger@seattlechildrens.org)
} 
a

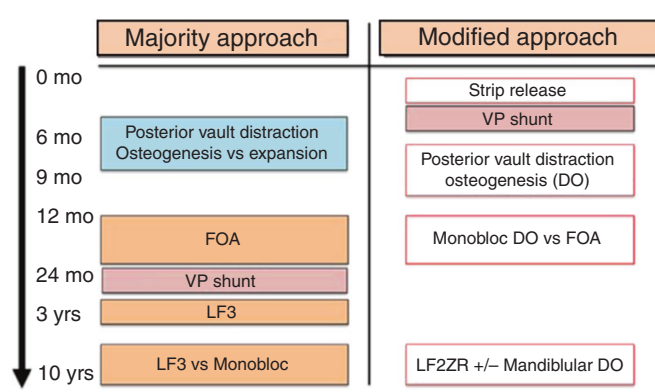

C
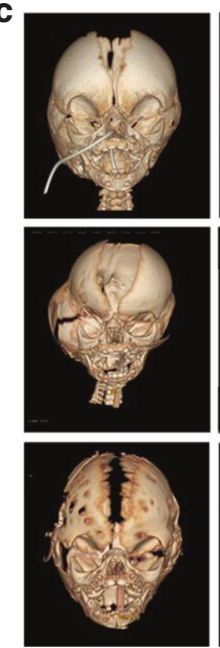
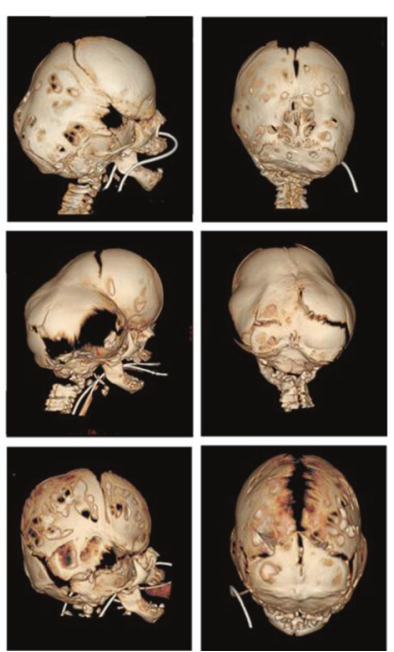
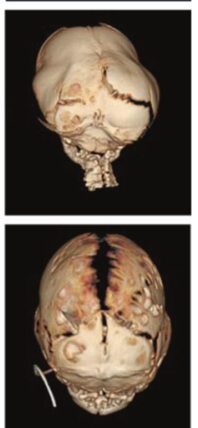

b

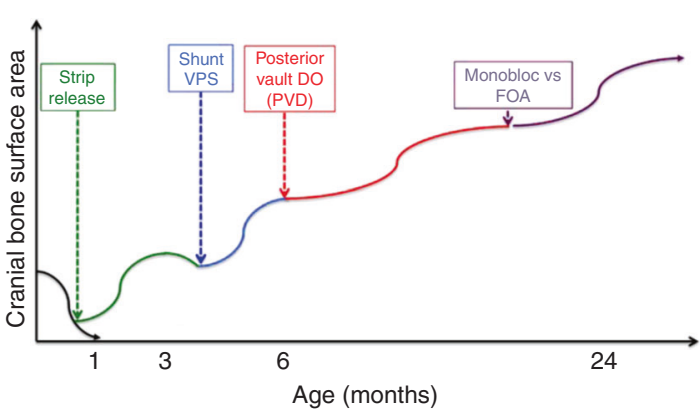

d
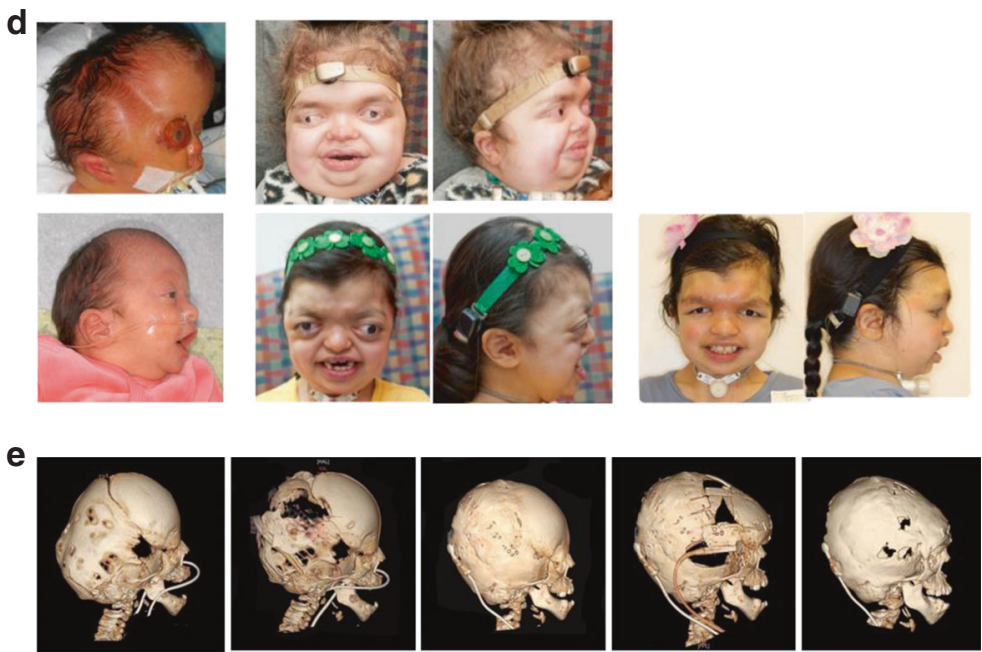

Fig. 1 Alternative and aggressive surgical protocol for severe craniosynostosis associated with FGFR2 pathogenic variant (PV). a Majority surgical approach for patients with Pfeiffer syndrome vs. modified alternative approach for children with severe posterior constriction, including all patients with W290C. FOA fronto-orbital advancement, VP ventriculoperitoneal, LF3 Le Fort 3 distraction osteogenesis, LF2ZR Le Fort 2 distraction osteogenesis with simultaneous zygomatic repositioning, DO distraction osteogenesis. b Bone-pressure wave illustrating changes in cranial bone surface area and timing of surgeries in modified Seattle approach. c Baseline head computed tomography (CT) scan with 3-D reconstruction for patient 1 (top), patient 2 (middle), and patient 3 (bottom) prior to surgical interventions. Note multisuture craniosynostosis and thinning of cranial bones. Views include AP (anterior-posterior, left), lateral (middle), and PA (posterior-anterior, right). d Photographs from patients 1 (bottom) and 2 (top) at similar ages for comparison of phenotype. First photograph (left) is prior to surgical intervention. Front and lateral view of patients at similar stage of surgical intervention at age 7-8 years for patient 2 (top) and patient 1 (bottom) shown in second and third columns. Note softband bone conduction sound processor in place for both patients. Patient 1 has additional photographs at age 11. Corresponding photographs not available for patient 1 as she is currently 7 years of age. e Progression of CT scans for patient 1 from infancy through age 10 as she progressed through surgical intervention

other needed medical procedures (e.g., some patients will need to have a tracheostomy placed or choanal stenosis repair prior to cranial vault expansion). The majority of patients with Pfeiffer at our center have required a common "majority approach" similar to that described in the literature at other centers. This involves posterior expansion at approximately 3-9 months followed by fronto-orbital advancement (along with possible Le Fort III $)^{18}$ or Monobloc advancement ${ }^{19,20}$ (Fig. 1a) and later suboccipital decompression. The timing of ventriculoperitoneal (VP) shunt placement, if necessary, is determined in each child by the development of hydrocephalus, but in our center often occurs around age 2 years for many children with Pfeiffer syndrome. Children who develop hydrocephalus before or after this time will receive a VPS when it is clinically indicated. While this majority protocol provides a general guideline for the surgical approach to Pfeiffer syndrome, each child is carefully evaluated for signs of worsening CCD or other symptoms that would suggest that earlier or more aggressive intervention is medically indicated.

Our high-volume craniofacial center served more than 150 patients with syndromic craniosynostosis in the past year, 30 of whom were new patients. All patients with multisuture craniosynostosis undergo the personalized pediatric and surgical evaluation with team consensus for timing and surgical readiness for the protocol as described above, and most will be treated according to the majority protocol, but others may require a modified approach in response to clinical and radiologic findings. Retrospective review of patients with multisuture craniosynostosis whose treatment differed from the majority approach protocol revealed (1) three patients who required an earlier and more aggressive surgical algorithm compared with the majority approach; (2) each of these three patients had the W290C PV in FGFR2; and (3) all three had excellent developmental outcomes, in stark contrast to those in the literature. 
We evaluated our cohort of children with Pfeiffer syndrome to detect associations between genotype, surgical approach, and clinical outcome. We focused on children with the W290C PV in FGFR2, whom we compared with published case reports of children with the same genotype. The purpose of this report is to describe the personalized surgical approach utilized in children with the W290C PV and to compare the developmental outcomes of our cohort of children with W290C PV who received the personalized and aggressive CCD approach, with those in the literature who had a less aggressive surgical approach.

\section{MATERIALS AND METHODS}

We performed a retrospective chart review of all patients with Pfeiffer syndrome seen at our center to evaluate the timing and nature of surgical procedures, genotype, and developmental level. We reviewed existing medical literature for comparison. Institutional review board (IRB) approval was obtained for medical chart review (IRB 00000167). Informed consent was obtained from the parents of all patients, including consent to publish patient photgraphs.

\section{RESULTS}

Personalized and aggressive approach to surgical management of cephalocranial disproportion

After reviewing medical records and existing literature, we found that our three patients with W290C PV in FGFR2 required a surgical approach characterized by earlier intervention and additional surgeries than would have occurred if they had been treated by the standard majority protocol (Table 1). All three children had comparable skull morphology on neonatal presentation with significant cranial constriction bands from vertex to sphenoid, with occipital constriction, compensatory frontal bossing and extensive cranial "thumbprinting" soon after birth. Neonatal computed tomography (CT) scans demonstrated lack of bone formation in compensating bossed areas around the constriction bands, consistent with pressure induced effacement. All three underwent a personalized surgical approach that was more aggressive and earlier than the other patients with Pfeiffer syndrome. These interventions were based on close monitoring of signs of CCD. These three patients were identified retrospectively by their deviation from the majority protocol, and then were subsequently identified by review of clinical molecular testing as being the three patients in our series with W290C PV. This retrospective association has now modified our approach so that early molecular identification of W290C $\mathrm{PV}$ is prioritized such that this information can be considered along with clinical characteristics in determining the appropriate surgical approach to optimize outcomes.

The personalized and aggressive CCD surgical approach starts with neonatal strip craniectomy to release constricting cranial bands in the first weeks of life. Improved cephalocranial disproportion is then assessed by subsequent bone formation in the previous areas of effacement. Early signs of hydrocephalus were treated with a ventriculoperitoneal shunt

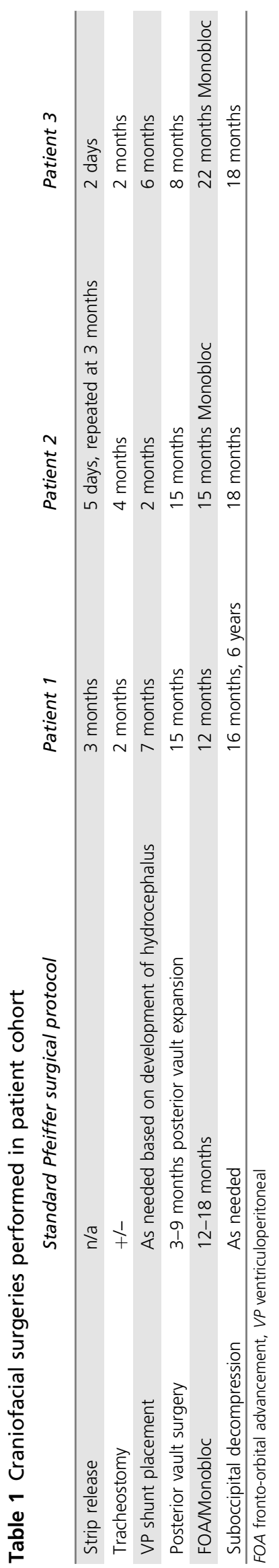


(VPS) placed in the first few months. Delay in VPS placement in these children will result in cephalocranial disproportion with loss of cranial bone surface area. With sufficient bone formation from early intervention, posterior vault distraction osteogenesis at 6-9 months of age, and either Monobloc advancement or frontal orbital advancement between 12 and 24 months of age, can be safely performed to achieve maximum intracranial expansion prior to 2 years of age. Subcranial Le Fort II with or without mandibular distraction osteogenesis was performed or is likely to be needed after the age of 7 for normalization of facial proportions and improved upper airway (Table 1). All three patients required suboccipital decompressions between the ages of 16 and 18 months. The oldest patient (patient 1) had a second suboccipital decompression at age 6 years. Due to this anticipated inevitable need for suboccipital decompression in patients with W290C, we now perform posterior fossa bone decompression at the same time as posterior vault distraction to minimize the number of surgeries. Each child also underwent tracheostomy to address tracheal cartilaginous sleeve and multilevel airway obstruction. Although tracheostomy is commonly needed in children with W290C PV this surgical approach is more intensive than what is described in existing literature.

The timing of these surgeries was based on the "bonepressure wave" concept that cranial release or expansion is performed to promote cranial bone deposition as a surrogate for intracranial homeostasis. See Fig. 1. The neonatal strip release was performed to stop progressive erosion of bone from the cranial bands. After release, the resulting cranial defects healed, but VPS placement for elevated intracranial pressure was required in all three children to treat elevated intracranial pressure (ICP) and avoid erosion of the newly deposited bone. After VPS placement, sufficient bone formed to provide the stability needed for a posterior vault expansion with distraction osteogenesis. This early and aggressive type of posterior cranial expansion then allowed frontal surgery to be performed well past the first year when stability of advancement is improved. Patients 2 and 3 underwent early Monobloc advancement to relieve upper airway obstruction as well as to treat severe exorbitism. Patient 1, our oldest patient, underwent frontoorbital advancement before early Monobloc was part of our treatment algorithm, and would have had that procedure if presenting today. As noted above, all three patients required suboccipital decompression with the oldest patient requiring a second suboccipital decompression.

\section{Growth}

All patients required nasogastric tube placement at birth, and have continued to require feeding via G-tubes. All three patients take a limited amount of food by mouth. All patients were born at average weight (patient 1: 30th percentile, patient 2: 25th percentile, patient 3: 35th percentile) and length (patient 1: 25th percentile, patient 2: 25th percentile, patient 3,35 th percentile). Head circumferences were difficult to accurately measure due to abnormal head shape at birth, and ranged widely in recorded size (patient 1: 25 th percentile, patient 2: 95th percentile, patient 3: 20th percentile). However, all have developed short stature. Patient 2 is currently at the 5th percentile, while patient 3 is 50th percentile for a 3 year old at 5 years of age and patient 1 is 50th percentile for a 7 year old at 11 years of age. Weight for length is normal for all patients. Head circumferences have also varied depending on stage of cranial vault expansion, with current percentiles in the normal range but disproportionately large compared to height (patient 1: 60th percentile, patient 2: 95th percentile, patient 3: 50th percentile).

\section{Respiratory support}

All patients received tracheostomy as infants and have never been decannulated. They all require positive pressure through their tracheostomies during sleep, but do not require any support while awake. Patient 1 uses a Passy-Muir valve during the day, and at night is on a Trilogy ventilator with synchronized intermittentmandatory ventilation (SIMV) pressure control plus pressure support (peak inspiratory pressure [PIP] 22, positive and expiratory pressure [PEEP] 5, pressure support 5 above PEEP, backup rate 18). Patient 2 requires bilevel positive airway pressure (BiPAP) during sleep (18/6 with a backup rate of 18). Patient 3 requires continuous positive airway pressure (CPAP) during sleep (pressure 6). The amount of positive pressure required during sleep has been titrated over time for all patients based on polysomnograms and they are closely followed by Sleep Medicine.

\section{Hearing}

Each of our patient has stenotic or atretic ear canals, which has resulted in conductive hearing loss. They have received regular audiograms and have no sensorineural hearing loss. Each patient is treated with a bone conduction sound processor, and they received special instruction in American Sign Language.

\section{Neurodevelopmental outcomes}

In contrast to previously reported patients, all three of our patients have excellent development. The first patient is 10 years old has completed grade 4 in a mainstream classroom setting with pullout for part of the day for children who are deaf or hard of hearing. She requires no academic support. She is working at grade level in math and above grade level in all other subjects. She has undergone statewide testing that is given to 4th graders across Washington State, and performed higher than the school, district, and state averages for both reading and math. The second patient is age 7 and is in a general education classroom with an aide to help with mobility and medical care. Because of physical challenges with speech production, she uses an iPad to help communicate, but is working at grade level, and her family and kindergarten teachers consider her to have normal intelligence. Formal neuropsychiatric assessments have not been completed. The third patient, age 4 , has not yet undergone formal assessment 
because of her physical limitations as well as the use of multiple languages used at home, including American Sign Language and Cambodian, and is demonstrating normal or near normal intelligence.

\section{DISCUSSION}

Patients with the W290C PV in FGFR2 are reported to have among the worst neurodevelopmental outcomes of any PV and a high risk of mortality. Surviving patients have historically had severely impaired neurocognitive functioning, as evidenced by a 15 year old who can follow simple commands and a 6 year old with a few signs who can sit unsupported. $^{10,12}$ Review of the literature reveals that all previously reported children who survived to the time of surgery underwent a treatment protocol comparable with what we would follow at our center for milder presentations; our so-called "majority approach." The clinical presentation of our patients at the time of birth was similar in the types and severity of medical problems of patients previously described in the literature. We propose that the superior developmental outcome in our patients is due to our modified surgical approach involving staged early and aggressive treatment of cephalocranial disproportion in the first 2 years of life.

While we cannot prove that this more intensive surgical approach was the reason for improved clinical outcome in our patients, we were unable to identify any other obvious differences in our patients compared with those in the literature. When photographs or imaging were available for previously reported patients, their skull morphology was comparable to our three patients. We hypothesize that the neurocognitive impairment in prior patients with Pfeiffer syndrome due to W90C PV is due to severe posterior skull constriction and secondary damage from increased intracranial pressure rather than a direct effect of the PV on the brain. Therefore, other PV that result in similar skull morphology could conceivably benefit from an early and aggressive surgical approach, though we are not aware of any other $\mathrm{PV}$ that have resulted in this specific phenotype. Conversely, if a patient with W290C PV were born without the typical posterior skull constriction that has been seen in all cases to date the "majority algorithm" might be sufficient. We have yet to encounter a patient with W290C in our center, however, who does not demonstrate early cephalocranial disproportion.

In addition to poor neurocognitive functioning, there has historically been a high rate of sudden death in infants with the W290C. This has been linked to airway occlusion in the setting of tracheal cartilaginous sleeves, which are estimated to confer a $90 \%$ risk of sudden death by age 2 without tracheostomy. ${ }^{12,14}$ All three of our patients received a tracheostomy in infancy due to multilevel airway obstruction, which likely contributed to their survival. Early Monobloc advancement, performed in patients 2 and 3, has only recently been described and is not yet practiced by many centers. ${ }^{19,20}$ The two families with a patient who underwent early Monobloc reported a dramatic improvement in nasopharyngeal and tracheostomy suctioning needs, which may have also contributed to an improved airway result. While we believe that early treatment of CCD likely had the largest effect on the excellent developmental outcomes in our patients, it is possible that the Monobloc advancement had an additional beneficial effect over anterior cranial expansion through the increase in upper airway volumes with this procedure. We recommend that early Monobloc should be considered in the treatment plan of other patients with W290C PV in FGFR2 or other PV with similar anatomic differences.

Although the associations between genotype, early and intensive surgical approach, and improved outcome were identified retrospectively, these cases suggest that prompt identification of patients with the W290C PV could allow for improved survival and development through a genotypeinfluenced treatment approach. Our group has previously suggested that early identification of W290C PV could allow for early operative airway evaluation and consideration of tracheostomy to prevent sudden death in patients with W290C-associated Pfeiffer syndrome. ${ }^{12}$ Our current data suggests that early and aggressive staged surgery to ameliorate occipital constriction and increased intracranial pressure in these patients could prevent the severe neurocognitive impairment that has been a feature of all previously reported children with this genotype.

\section{Conclusions}

In contrast to existing literature, the three children with W290C PV in FGFR2 presented here had excellent clinical outcomes. This leads us to conclude that poor outcomes are not an inevitable consequence of the W290C genotype, and prognosis may be improved by a genotype-influenced early and aggressive medical and surgical approach. Early genetic testing of infants with suspected Pfeiffer syndrome may identify infants with W290C PV and allow consideration of early tracheostomy and a more intensive craniofacial surgical approach to optimize developmental outcome. Geneticists and neonatologists should make sure these infants are evaluated in the neonatal period by a craniofacial team experienced in surgical management of patients with multisuture craniosynostosis.

These data also provide additional support for the medical necessity of genetic testing for patients with suspected Pfeiffer syndrome as patients with the W290C PV may benefit from a more intensive medical and surgical approach to prevent sudden death and optimize long-term developmental outcomes. Furthermore, families of children affected by the W290C PV which is historically associated with a dismal prognosis, should be aware that excellent clinical outcomes are now achievable.

\section{ACKNOWLEDGEMENTS}

We would like to acknowledge Erik Stuhaug for patient photography, as well as the patients and families for their participation. 


\section{FUNDING}

There are no relevant funding sources for this manuscript.

\section{DISCLOSURE}

The authors declare no conflicts of interest.

\section{REFERENCES}

1. Ariga $H$, Endo $Y$, Ujiie N, et al. Trp290Cys mutation of the FGFR2 gene in a patient with severe Pfeiffer syndrome type 2. Pediatr Int 2001;43:293-5.

2. Barry GP, Ny BM-L, Zackai EH, et al. A case report of a patient with Pfeiffer syndrome, an FGRF 2 mutation (Trp290Cys) and unique ocular anterior segment findings. Ophthalmic Genet. 2010;31:193-5.

3. Chen CP, Lin SP, Su YN, et al. Craniosynostosis and congenital tracheal anomalies in an infant with Pfeiffer syndrome carrying the W290C FGFR2 mutation. Genet Couns. 2008;19:165-72.

4. Ettinger N, Williams M, Phillips JA. Variable expressivity and clinical heterogeneity can complicate the diagnosis and management of Pfeiffer syndrome. J Craniofac Surg. 2013;24:1829-32.

5. Hockstein NG, McDonald-McGinn DM, Zackai EH, et al. Tracheal anomalies in Pfeiffer syndrome. Arch Otolaryngol Neck Surg. 2004;130:1298.

6. Lajeunie E, Heuertz S, El Ghouzzi V, et al. Mutation screening in patients with syndromic craniosynostoses indicates that a limited number of recurrent FGFR2 mutations accounts for severe forms of Pfeiffer syndrome. Eur J Hum Genet. 2006;14:289-98.

7. Nazzaro A, Della Monica M, Lonardo F, et al. Prenatal ultrasound diagnosis of a case of Pfeiffer syndrome without cloverleaf skull and review of the literature. Prenat Diagn. 2004;24:918-22.

8. Oliveira NAJ, Alonso LG, Fanganiello RD, et al. Further evidence of association between mutations in FGFR2 and syndromic craniosynostosis with sacrococcygeal eversion. Birth Defects Res A Clin Mol Teratol. 2006;76:629-33.
9. Schaefer F, Anderson C, Can B, et al. Novel mutation in the FGFR2 gene at the same codon as the Crouzon syndrome mutations in a severe Pfeiffer syndrome type 2 case. Am J Med Genet. 1998;75:252-5.

10. Shotelersuk V, Ittiwut C, Srivuthana S, et al. Distinct craniofacial-skeletaldermatological dysplasia in a patient with W290C mutation in FGFR2. Am J Med Genet. 2002;113:4-8.

11. Tartaglia M, Valeri S, Velardi F, et al. Trp290Cys mutation in exon Illa of the fibroblast growth factor receptor 2 (FGFR2) gene is associated with Pfeiffer syndrome. Hum Genet. 1997;99:602-6.

12. Wenger TL, Dahl J, Bhoj EJ. Tracheal cartilaginous sleeves in children with syndromic craniosynostosis. Genet Med. 2017;19:62-8.

13. Zackai EH, McDonald-McGinn DM, Stolle C, et al. Craniosynostosis with tracheal sleeve: a patient with Pfeiffer syndrome, tracheal sleeve and additional malformations in whom an FGFR2 mutation was found. Clin Dysmorphol. 2003;12:209.

14. Lertsburapa K, Schroeder JW, Sullivan C., et al. Tracheal cartilaginous sleeve in patients with craniosynostosis syndromes: a meta-analysis. J Pediatr Surg. 2010;45:1438-44.

15. Sandler AL, Goodrich JT, Daniels LB, et al. Craniocerebral disproportion: a topical review and proposal toward a new definition, diagnosis, and treatment protocol. Childs Nerv Syst. 2013;29:1997-2010.

16. McCarthy JG, Glasberg SB, Cutting CB, et al. Twenty-year experience with early surgery for craniosynostosis: II. The craniofacial synostosis syndromes and pansynostosis-results and unsolved problems. Plast Reconstr Surg. 1995;96:284-95. Discussion 296-8.

17. White N, Evans M, Dover MS, et al. Posterior calvarial vault expansion using distraction osteogenesis. Childs Nerv Syst. 2009;25:231-6.

18. Arnaud E, Paternoster $G$, James $S$, et al. Stratégie craniofaciale pour les faciocraniosténoses. Ann Chir Plast Esthét. 2016;61:408-19.

19. Ahmad F, Cobb AR, Mills C, et al. Frontofacial monobloc distraction in the very young: a review of 12 consecutive cases. Plast Reconstr Surg. 2012;129:488e-97e.

20. Visser R, Ruff CF, Angullia F, et al. Evaluating the efficacy of Monobloc distraction in the Crouzon-Pfeiffer craniofacial deformity using geometric morphometrics. Plast Reconstr Surg. 2017;139:477e-87e. 